\title{
Surface phosphorylation of chitosan significantly improves osteoblast cell viability, attachment and proliferation $\dagger$
}

\author{
Paula M. López-Pérez, ${ }^{* a b}$ Ricardo M. P. da Silva, ${ }^{a b}$ Carmen Serra, ${ }^{c}$ Iva Pashkuleva ${ }^{* a b}$ and Rui L. Reis ${ }^{a b}$
}

\author{
Received 17th June 2009, Accepted 27th October 2009 \\ First published as an Advance Article on the web 19th November 2009 \\ DOI: $10.1039 / b 911854 c$
}

Chitosan biocompatibility is often associated with the structural similarities with glycosaminoglycans (GAGs). Although all of the GAGs are built from repeating disaccharide units and some of them contain $\mathrm{N}$-glucosamine (the main hexosamine in the chitosan backbone), all of them also contain negatively charged functional groups. These charged units are believed to have a crucial role for the formation of proteoglycans and hence for key biochemical processes/signaling related to cell functionality and survival. Lack of these groups in chitosan structure could be the reason for the previously observed poor cell adhesion to this material. Herein, we report that plasma induced grafting of negatively charged phosphonic groups can induce remarkably distinguishable cell response and significantly improve the adhesion, proliferation and viability of osteoblast cells. The proposed plasma induced polymerization is a very simple and versatile method and can be easily adapted to other materials and different negatively charged units.

\section{Introduction}

The presence of complex polysaccharides in different tissues has been recognized for more than 100 years. In connective tissues, e.g. cartilage, they comprise about $30 \mathrm{wt}^{\%} .{ }^{1}$ Some polysaccharides represent the main constituent part of the extracellular matrix (ECM). Hence, they are involved in a plethora of biological processes and thus they are crucial in the control of their normal metabolic course. Structural investigations revealed similarities between these polysaccharides: ${ }^{1}$ they are composed of disaccharide repeating units, they all contain hexosamine (glucosamine or galactosamine), and finally they do contain negative functionalities (mostly carboxyl and sulfate). Their common name, glycosaminoglycans (GAGs), comprises these similarities.

Chitosan, a linear polysaccharide composed of $N$-glucosamine and $N$-acetylglucosamine units, has been accepted in the biomaterials field as a structural analogue of GAGs. Its structure together with its relatively low price has imposed an intensive investigation on this polysaccharide as a material for a wide range of biomedical applications, such as wound healing, gene/ drug delivery, bone tissue repair and remodeling. ${ }^{2,3}$ Furthermore, its biodegradation leads to the release of aminosugars that can easily be excreted or incorporated into glycoproteins and GAGs metabolic pathways. ${ }^{4}$

a3B's Research Group - Biomaterials, Biodegradables and Biomimetics, University of Minho Headquarters of the European Institute of Excellence on Tissue Engineering and Regenerative Medicine, AvePark, 4806-909 Taipas, Guimarães, Portugal._E-mail: plopez@dep.uminho.pt; ricardosilva@dep.uminho.pt; pashkuleva@dep.uminho.pt; rgreis@dep. uminho.pt; Fax: + 351253 510909; Tel: + 351253510900

${ }^{b} I B B$ - Institute for Biotechnology and Bioengineering, PT Government Associated Laboratory, Braga, Portugal

'University of Vigo, CACTI, E-36310, Vigo, Spain. E-mail: cserra@, uvigo.es

$\dagger$ Electronic supplementary information (ESI) available: Fig. S1, S2 and Table S1. See DOI: 10.1039/b911854c
Besides these similarities, the $\mathrm{p} K_{\mathrm{a}}$ value of chitosan is about $6.1-7^{5}$ and recently it was reported ${ }^{6}$ that the chitosan membrane isoelectric point (zeta potential equal to zero) occurs under physiological conditions ( $\mathrm{pH}$ 7.4). Hence, the positive surface charge arising from protonated amino groups, often claimed to predict cell adhesion to chitosan, should be excluded. Because the negative charge of the GAGs is associated with their bioactivity (via interactions with the positively charged amino groups of proteins), the lack of these groups in chitosan could be the reason for the poor cell adhesion on chitosan membranes reported previously by us ${ }^{7,8}$ and other authors. ${ }^{9-11}$ Several studies have been investigating the possibility to overcome this major drawback by incorporation of carboxyl or sulfate groups into the chitosan backbone mimicking GAGs structure. ${ }^{6,712,13}$ However, when regeneration of mineralized tissues such as bone is the target, in addition to the protein-polysaccharide interactions, osseointegration and osteoconduction are also a must. These processes are triggered by the presence of other negatively charged groups, the phosphate groups. The importance of these groups has been recognized by the biomaterials scientific community for a long time, and nowadays phosphate coated (e.g. calcium phosphate) materials are commonly used for the purposes of bone regenerative medicine. Alternatively, polymers with grafted phosphate groups have also been designed to mimic the interactions occurring in vivo. In contrast to physically coated polymers, the grafting process results in the formation of covalent bonds between the graft chains/groups and the polymer surface and therefore avoids their delamination, assuring long term stability of the introduced chains. The fundamental step in grafting is the formation of reactive groups on the substrate surface. So far, only chemical activation/functionalization has been reported for obtaining phosphorylated derivatives of chitosan. ${ }^{14-20}$ These reactions usually involve the use of organic solvents and high temperatures and therefore can easily affect the bulk properties (e.g. mechanical properties, degradation behavior) of the material. Hence, we propose an alternative 
approach for surface phosphorylation of chitosan membranes by plasma induced polymerization. Previously, we have demonstrated $^{7}$ that this method is an effective way for the grafting of vinyl monomers. Its main advantage is that the surface properties can be enhanced selectively, while the bulk attributes of the materials remain unchanged. In this particular study, we report the use of vinyl phosphonic acid (VPA) as a monomer. Polymers of VPA are known to be non-cytotoxic and have been successfully included onto polymers structures for cell behavior enhancement. $^{21,22}$ It should be also noted that the herein proposed method for surface phosphorylation does not remove the amino groups from the chitosan surface. The preservation of these distinct functionalities in chitosan is very important because they are associated with the specific biological properties of chitosan. ${ }^{6}$

\section{Materials and methods}

\section{Materials}

Chitosan (CTS) from crabs' shells was purchased from Sigma Aldrich and purified prior use. Briefly, a 1\% (w/v) chitosan solution in $1 \%$ aqueous acetic acid $(\mathrm{AcOH})$ solution was prepared and filtered in order to remove the insoluble impurities. Subsequently, chitosan was coagulated by adding $\mathrm{NaOH}(0.1 \mathrm{M})$ solution (final $\mathrm{pH}>8$ ) and the formed gel was washed with distilled water until a stable $\mathrm{pH}$ was reached. The obtained product was dehydrated by immersion in absolute ethanol, freeze-dried, ground to a powder and dried overnight at $60{ }^{\circ} \mathrm{C}$.

The degree of $N$-deacetylation (DD) of chitosan (93\%) was determined by first derivative UV spectrophotometry, using both glucosamine (GluN) and $N$-acetylglucosamine (GluNAc) as standards for calibration. ${ }^{23}$ A molecular weight of $790 \mathrm{kDa}$ was determined by viscometry using a $0.5 \mathrm{M} \mathrm{AcOH}-0.2 \mathrm{M} \mathrm{AcONa}$ aqueous solution. The measurements were performed at $25^{\circ} \mathrm{C}$ and the Mark-Houwink parameters $\left(k=3.5 \times 10^{-4} ; a=0.76\right)^{24}$ were used for the calculations. Vinyl phosphonic acid (VPA) was purchased from Sigma-Aldrich and was used without further purification.

\section{Preparation and modification of chitosan membranes}

Chitosan $1 \%(\mathrm{w} / \mathrm{v})$ was dissolved in $1 \%$ aqueous $\mathrm{AcOH}$ with slow stirring to avoid air bubble entrapment. The chitosan solution was poured into plastic Petri dishes at $0.5 \mathrm{mg} \mathrm{cm}^{-2}$. After drying in air, the membranes were neutralized by immersion in $0.1 \mathrm{M}$ $\mathrm{NaOH}$ for $10 \mathrm{~min}$ and washed thoroughly with distilled water. The obtained membranes presented a smooth surface and thickness of around $50 \mu \mathrm{m}$.

Poly(phosphonic acid) PVPA was grafted on the membranes surface by plasma induced polymerization. Chitosan membranes were placed into a radio frequency $(13.56 \mathrm{MHz})$ plasma reactor (Plasma Prep5 equipment from Gala Instrument, Germany) and exposed to oxygen plasma at $30 \mathrm{~W}$ of power for $15 \mathrm{~min}$. During the treatment the pressure inside the reactor was maintained below $20 \mathrm{~Pa}$ by adjusting the gas flow. The so-activated membranes with free radicals formed on the surface were immersed in $100 \mathrm{mM}$ degassed solution of VPA in isopropanol and shaken at $37^{\circ} \mathrm{C}$ for $2 \mathrm{~h}$. Subsequently, the membranes were thoroughly washed with isopropanol to remove the unreacted monomer and dried at room temperature.

\section{Surface characterization}

Surface chemical composition. Surface elemental analyses of non-treated and modified samples was performed by X-ray photoelectron spectroscopy (XPS) using an ESCALAB 200A instrument (VG Scientific, UK) with an aluminium anode (Al-K $\alpha$ monochromatic radiation $h \nu=1486.6 \mathrm{eV}$ ) operating at $15 \mathrm{kV}(300 \mathrm{~W})$. The measurements were performed at a take off angle of $90^{\circ}$ relative to the samples surface and a constant Analyser Energy mode (CAE). PISCES software was used for data acquisition and analysis. Survey spectra were acquired using a pass energy of $50 \mathrm{eV}$ over a binding energy range of 0 to $1100 \mathrm{eV}$ and were used to calculate the elemental composition of the surfaces. High-resolution spectra for different elements $(\mathrm{C} 1 \mathrm{~s}$, $\mathrm{O} 1 \mathrm{~s}$ and $\mathrm{P} 2 \mathrm{p}$ ) were obtained using a pass energy of $20 \mathrm{eV}$. Deconvolution into subpeaks was performed by least-squares peak analysis software, XPSPEAK version 4.1, using the Gaussian/Lorenzian sum function. Background counts were subtracted using a linear baseline and the sample charging was corrected by assigning a binding energy of $285.0 \mathrm{eV}$ to the saturated hydrocarbons $\mathrm{C} 1 \mathrm{~s}$ peak.

Time-of-flight secondary ion mass spectrometry (ToF-SIMS) studies were performed using a ToF-SIMS IV instrument from ION-TOF GmbH, Germany. ToF-SIMS produces positive and negative mass spectra from the outer 10-20 $\AA$ of materials, and thus is capable of providing detailed information about the molecular structure of surfaces. The sputtering process is central to the SIMS technique and it can be described as a collision cascade of particles in the sample being analyzed. In this study, the samples were bombarded with a pulsed bismuth ion beam $(25 \mathrm{keV})$ at $45^{\circ}$ incidence over an area with size $500 \mu \mathrm{m}^{2}$. The energy of these primary ions is enough for bond breaking near to the collision site and therefore, the process results in extensive fragmentation and emission of secondary particles (neutral atoms and molecules, electrons, and ions). Particles produced in the top 2-3 monolayers of the sample have sufficient energy to overcome the surface binding energy and leave the sample. Only a small fraction of them are charged $\left(10^{-6}\right.$ to $\left.10^{-1}\right)$, and their positive or negative state depends on their electron configuration. The generated secondary ions were extracted with a voltage of $10 \mathrm{kV}$ and their mass was determined by measuring their time-offlight from the sample to the detector. An electron flood gun for charge compensation was necessary during the measurements. The experimental conditions (ion type, beam voltage and primary ion dose) were maintained constant for comparative studies. The mass spectra in both positive and negative mode and some specific informative secondary ion images for treated and modified samples are reported in the following section. Additionally, high mass resolution spectra were obtained by bunching the raw pulse. These spectra can be attained without concurrent loss of counts, however, this is at the cost of spatial resolution (in this mode no better than $2-5 \mu \mathrm{m}$ ).

Surface topography. The topography of the samples was characterized by two different techniques: optical profiler analysis was performed using an Interferometric profiler Wyko-NT 
1100 (Veeco) using vertical scanning interferometry (VSI) mode. The images were processed and analyzed with the analytical software package WycoVision ${ }^{\circledR} 32$. The atomic force microscopy (AFM) characterization was performed in air using a Multimode Nanoscope V (Veeco). Tapping mode was employed with a noncoated phosphorous (n)-doped silicon probe with cantilever length of 115 to $135 \mu \mathrm{m}$ and resonant frequency from 257 to $342 \mathrm{kHz}$. Images were processed and analyzed using multimode software version V7.20 and analytical software package WycoVision ${ }^{\circledR} 32$.

Surface energy and water wettability. Static contact angle values were obtained using the sessile drop method. Measurements were performed using a contact angle meter OCA 15+ (DataPhysics Instruments, Germany) with a high performance image processing system. A $1 \mu \mathrm{l}$ drop of the tested liquid was added at room temperature onto the sample surface by a motor driven syringe. We used the three-solvent system: water $\left(\gamma_{\mathrm{s}}=\right.$ $\left.72.8 \mathrm{mN} \mathrm{m}^{-1}\right)$ and glycerol $\left(\gamma_{\mathrm{s}}=64.0 \mathrm{mN} \mathrm{m}^{-1}\right)$ as polar liquids and methylene iodide $\left(\gamma_{\mathrm{s}}=50.8 \mathrm{mN} \mathrm{m}^{-1}\right)$ as the non-polar one. At least six contact angle replicates per liquid-solid couple were measured and averaged. We calculated the surface tension from the contact angle data by two different methods: the Owens, Wendt, Rabel and Kaelble (OWRK) method ${ }^{25}$ that discerns polar and dispersive components of the surface energy; and the acid-base method (AB method), which allows the calculation of the Lewis acid and basic contributions using the van OssChaudury-Good theory (vOCG). ${ }^{26}$

The chosen three-solvent system can be used to compute the surface energy using the vOCG method, since it produces a set of well conditioned equation systems (low conditioning number). ${ }^{27}$ The surface energy components were calculated using the values of the liquid surface tension components obtained by Della Volpe and Siboni, which consider a reference scale for water that takes into consideration the different "strength" of water as acid or base. ${ }^{28}$

Pure water adhesion tension was also calculated because it can be considered as a key measure of the water self-association structure. As a matter of fact, a straightforward correspondence has been claimed to occur between the physicochemical interfacial properties of water (scaled as water adhesion tension) and the biological response to biomaterials. ${ }^{29}$

\section{Cell culture}

Since the materials studied in this work are to be used for mineralized tissue regeneration, the effect of the surface modifications was assessed by cells with the osteoblastic phenotype, the principal cell type facing these devices in vivo. A human osteosarcoma cell line (SaOs-2), an immortalized cell line with an osteoblastic phenotype, was purchased from European Collection of Cell Cultures (ECACC, UK) and cells were maintained at $37{ }^{\circ} \mathrm{C}$ and $5 \% \mathrm{CO}_{2}$ in a humidified atmosphere. Dulbecoo's modified Eagle's medium (DMEM; Sigma-Aldrich, Inc, USA) supplemented with $10000 \mathrm{U} \mathrm{ml}^{-1}$ penicillin-G sodium, $10000 \mu \mathrm{g}$ $\mathrm{ml}^{-1}$ streptomycin sulfate and $25 \mu \mathrm{g} \mathrm{ml}^{-1}$ amphotericin $\mathrm{B}$ in a $0.85 \%$ saline (Gibco, Invitrogen Corporation, UK) and $10 \%$ of heat-inactivated fetal bovine serum (FBS; Biochrom AG, Germany) were used as the cell culture medium.
Membranes were cut into a circular shape $(\phi=14 \mathrm{~mm})$ and were sterilized using a $70 \%$ ethanol aqueous solution. Subsequently, the substrates were washed with sterile phosphate buffered saline solution (PBS, Sigma Chemical Co., USA) to remove the remaining ethanol. The sterile samples were placed into 24 well culture plates and seeded with SaOs-2 $\left(3.3 \times 10^{4}\right.$ cells per $\mathrm{ml}$ ). Cells were cultured onto the materials for 1, 3, 7 and 14 days at $37{ }^{\circ} \mathrm{C}, 5 \% \mathrm{CO}_{2}$ in a humidified atmosphere in order to follow their behavior in contact with the studied surfaces.

The morphology of SaOs-2 cells growing on untreated and modified chitosan membranes was observed by scanning electron microscopy (S360, Leica Cambridge, UK). After each predetermined incubation time, cells were fixed using a $2.5 \%$ glutaraldehyde (Sigma, USA) solution in PBS. Prior to the analysis, the samples were dehydrated by graded ethanol solutions $(25 \%, 50 \%, 70 \%, 90 \%$ and $100 \%)$.

MTS assay and calcein-AM (MolecularProbes) staining were used to analyze cell viability. The cultured materials were incubated $\left(3 \mathrm{~h}, 37^{\circ} \mathrm{C}\right.$, humidified atmosphere of $\left.5 \% \mathrm{CO}_{2}\right)$ with $500 \mu \mathrm{l}$ of MTS solution in DMEM culture medium without phenol red (Sigma-Aldrich, Inc, USA). Optical Density (OD) was read in a microplate reader (Bio-Tek, USA) at $490 \mathrm{~nm}$. For Calcein AM staining, the samples were treated with $0.002 \%$ calcein-AM solution in DMEM culture medium and incubated in the dark for $15 \mathrm{~min}$ at $37^{\circ} \mathrm{C}$ in a humidified atmosphere of $5 \% \mathrm{CO}_{2}$. Cell fluorescence was examined using an Axioplan Imager $\mathrm{Z} 1$ from Zeiss, Germany.

Cell proliferation was evaluated by DNA quantification. Cells were lysed by osmotic and thermal shock and the obtained supernatant was used for DNA analysis. DNA content along the time of culture using the PicoGreen dsDNA kit (MolecularProbes) and the fluorescence was read $(485 \mathrm{~nm} / 528 \mathrm{~nm}$ of excitation/emission) using a microplate reader and the DNA amounts calculated from a standard curve.

The MTS and DNA quantification data were subjected to statistical analysis and are reported as mean \pm standard deviation. ANOVA tests for independent samples were performed and the differences were considered statistically significant if $p<0.05$.

\section{Results and discussion}

\section{Surface chemistry}

The XPS spectrum of untreated chitosan membranes confirms the presence of $\mathrm{C}(63.2 \%), \mathrm{O}(27.3 \%)$ and $\mathrm{N}(5.1 \%)$. Although at lower percentage, $\mathrm{Si}(4.3 \%)$ was also detected in the atomic composition of the chitosan surface. Silicon is a component of crustacean shells, from which chitin is extracted and then converted into chitosan and it seems to be not completely removed during that process. After the applied surface treatment, the survey spectrum of PVPA grafted membranes showed two additional peaks (see ESI $\dagger$ ) at 128.3 and $185.2 \mathrm{eV}$. These peaks were assigned to $\mathrm{P} 2 \mathrm{p}$ and $\mathrm{P} 2 \mathrm{~s}$, respectively, and confirmed the successful grafting with PVPA, incorporating 2 atomic $\%$ (at $\%$ ) of phosphorous (see ESI $\dagger$ ). Additional details about the surface chemical composition were obtained by high resolution spectra of $\mathrm{C} 1 \mathrm{~s}, \mathrm{O} 1 \mathrm{~s}$ and P2p of unmodified and PVPA grafted chitosan membranes (Fig. 1). The corresponding relative peaks are listed in Table 1. 

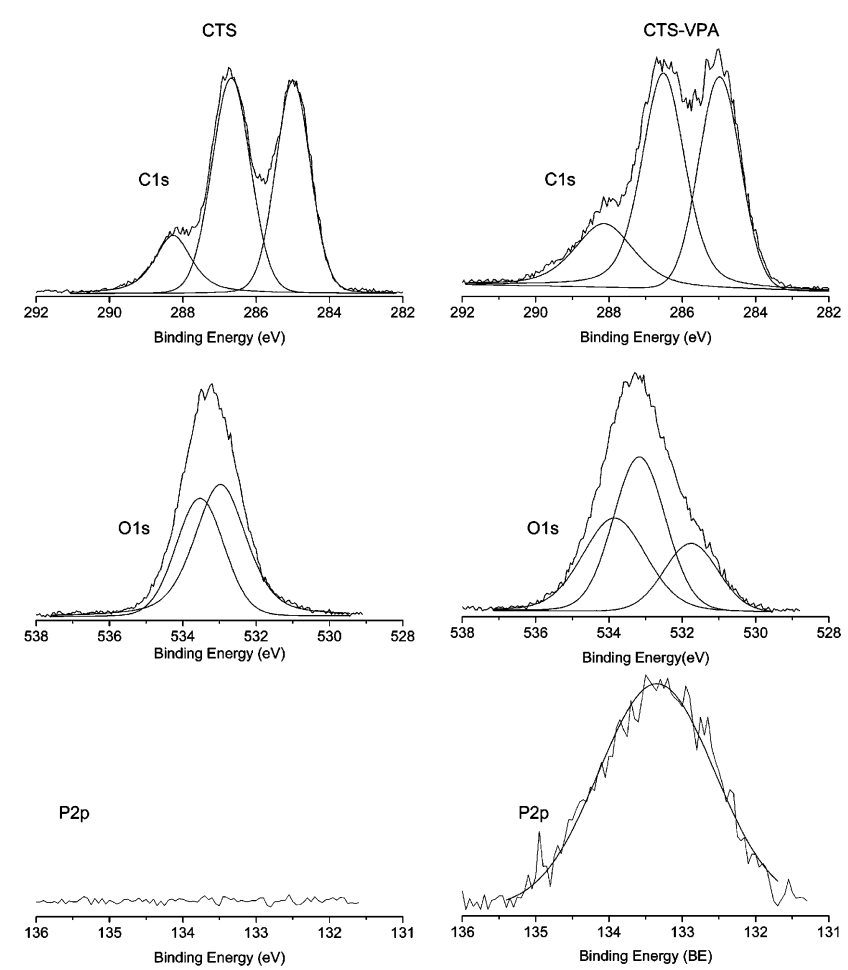

Fig. $1 \mathrm{C} 1 \mathrm{~s}, \mathrm{O} 1 \mathrm{~s}, \mathrm{P} 2 \mathrm{p}$ core level spectra of native and PVPA grafted chitosan membranes.

Table 1 Relative peaks composition (\%) of $\mathrm{C} 1 \mathrm{~s}$, O1s high resolution spectra

\begin{tabular}{lllllll}
\hline C1s core level & & & & \multicolumn{2}{l}{ O1s core level } \\
\cline { 1 - 3 } \cline { 5 - 6 } Peak BE/eV & CTS & CTS-VPA & & Peak BE/eV & CTS & CTS-VPA \\
\hline 285.0 & 40.5 & 36.6 & & 531.8 & 0.0 & 20.2 \\
286.6 & 44.6 & 44.4 & & 533.1 & 58.2 & 45.1 \\
288.2 & 14.9 & 19.0 & & 533.7 & 41.8 & 34.6 \\
\hline
\end{tabular}

The C1s high resolution spectrum of chitosan is composed of three components. The peak at $285.0 \mathrm{eV}$ was assigned to $C-\mathrm{H} / C-$ $\mathrm{C}$ chemical bonds of the chitosan backbone. Because the presence of amines induces a small chemical shift (around $0.6 \mathrm{eV}$ ), ${ }^{30}$ the $C-\mathrm{NH}_{2}$ signal does not appear as an individual peak but it is overlapped by the $\mathrm{C}-\mathrm{H} / \mathrm{C}-\mathrm{C}$. The second peak is centered at $286.7 \mathrm{eV}$ and was assigned to $C-\mathrm{OH}, C-\mathrm{O}$ and $C-\mathrm{N}$ bonds. Finally, the $\mathrm{C}=\mathrm{O}$ and $\mathrm{O}-\mathrm{C}-\mathrm{O} / \mathrm{N}-\mathrm{C}=\mathrm{O}$ groups from the acetylated rings appeared at $288.2 \mathrm{eV}$. The $\mathrm{C} 1 \mathrm{~s}$ high resolution spectrum of PVPA grafted samples was very similar to the spectrum of untreated chitosan. The characteristic $C-\mathrm{PO}_{3}$ signal (around $287.5 \mathrm{eV}^{31}$ ) is overlapped by the peak centered at 288.1 $\mathrm{eV}$ and the increased relative area measured for this peak compared with untreated chitosan (Table 1) is consistent with this overlapping. The O1s high resolution spectrum of chitosan showed two peaks centered at 533.0 and $533.5 \mathrm{eV}$, which correspond to $\mathrm{C}-\mathrm{OH}$ and $\mathrm{O}-\mathrm{C}-\mathrm{O}$ bonds, respectively. ${ }^{16}$ After PVPA grafting a new signal at $531.8 \mathrm{eV}$ was observed in the $\mathrm{O} 1 \mathrm{~s}$ spectrum, assigned to $\mathrm{O}$ atoms involved in $-\mathrm{PO}_{3}$ bonds. ${ }^{32}$ Considering the presence of elemental $\mathrm{P}$ on the surface $(2 \mathrm{at} \%)$ and the fact that three $\mathrm{O}$ atoms are bound to each $\mathrm{P}$ atom $\left(-\mathrm{PO}_{3}\right)$, the expected phosphorous-bound oxygen should be $6 \%$ of the total elemental composition on the surface. The deconvolution of the $\mathrm{O} 1 \mathrm{~s}$ high resolution spectrum gave a relative oxygen abundance in the $-\mathrm{PO}_{3}$ of $20.2 \%$ (Table 1). Because the total surface content of oxygen was found to be $32.1 \mathrm{at} \%$ (see ESI $\dagger$ ), the final experimental value for elemental phosphorous-bound oxygen is calculated to be $6.4 \%$ of the total elemental composition on the surface, which is in good agreement with the theoretical value. Finally, the P2p high resolution spectrum for PVPA grafted samples showed a single peak centered at $133.3 \mathrm{eV}$, assigned to the phosphonic species. ${ }^{33-35}$ The observed full width at halfmaximum (FWHM) for this signal is wide $(1.86 \mathrm{eV})$, as can be expected for $\mathrm{P} 2 \mathrm{p}$, since this signal corresponds to $\mathrm{P} 2 \mathrm{p}_{1 / 2}$ and $\mathrm{P} 2 \mathrm{p}_{3 / 2}$ core-line doublets.

In addition to XPS, ToF-SIMS analysis is used to identify the chemical and molecular composition of a surface. Small variations in the samples can be detected by differences in the fragmentation pattern in the mass spectra. Fig. 2 displays both positive and negative ion survey spectra of the chitosan surface prior to and after the applied modification. Chitosan is composed of randomly distributed D-glucosamine (molecular mass 179) and $N$-acetyl-D-glucosamine (molecular mass 221) units. Hence, fragmentation patterns observed in the mass spectra of these glucosamines were also expected in the chitosan spectrum. Indeed, the ions M18 $\left(\mathrm{NH}_{4}\right), \mathrm{M} 30\left(\mathrm{CH}_{4} \mathrm{~N}\right), \mathrm{M} 59$ $\left(\mathrm{C}_{2} \mathrm{H}_{5} \mathrm{NO}\right), \quad \mathrm{M} 73\left(\mathrm{C}_{3} \mathrm{H}_{7} \mathrm{NO}\right)$, and the characteristic M207 $\left(\mathrm{C}_{7} \mathrm{H}_{13} \mathrm{NO}_{6}\right)$ and $\mathrm{M} 221\left(\mathrm{C}_{8} \mathrm{H}_{15} \mathrm{NO}_{6}\right)^{36}$ were detected in the positive ToF-SIMS spectra of chitosan (modified and nonmodified). After PVPA grafting, we were not able to detect the expected M31 (P) and M15 (NH) in positive polarity. However, it must be stated that the $\mathrm{P}$ signal at this polarity presents a very low intensity and therefore it is rather difficult to detect. On the other hand, the negative spectrum of PVPA-grafted chitosan clearly showed the presence of the specific fragments at M63 $\left(\mathrm{PO}_{2}\right)$ and $\mathrm{M} 79\left(\mathrm{PO}_{3}\right)$. Additionally, the high resolution ToFSIMS (Fig. 3) also confirmed the presence of phosphorus with the characteristic peak at 30.975 present only in the spectrum of the modified material. Moreover, a much higher concentration of M15 (NH) was detected after plasma modification demonstrating the simultaneous occurrance of both processes grafting on one hand and etching/cleaning of the surface during the plasma activation on the other hand.

Further investigation of the spatial distribution of the new functionalities introduced by the applied modification was performed by ToF-SIMS mapping (Fig. 4). The mapping confirmed that the grafting was successful and phosphorous containing groups at high concentration (brightest regions) were clearly observed on the overall surface. Although, it should be noted that the modified surface was not chemically homogeneous and some regions with lower concentration of $\mathrm{PO}_{2}$ and $\mathrm{PO}_{3}$ fragments were observed.

\section{Surface topography}

In theory, surface modifications can be envisaged to vary independently to the surface topography and chemistry. However, in practice this is very difficult to achieve and in fact it is impossible to prove that both properties are independent. ${ }^{37}$ Hence, surfaces subjected to chemical modifications should be also evaluated for 

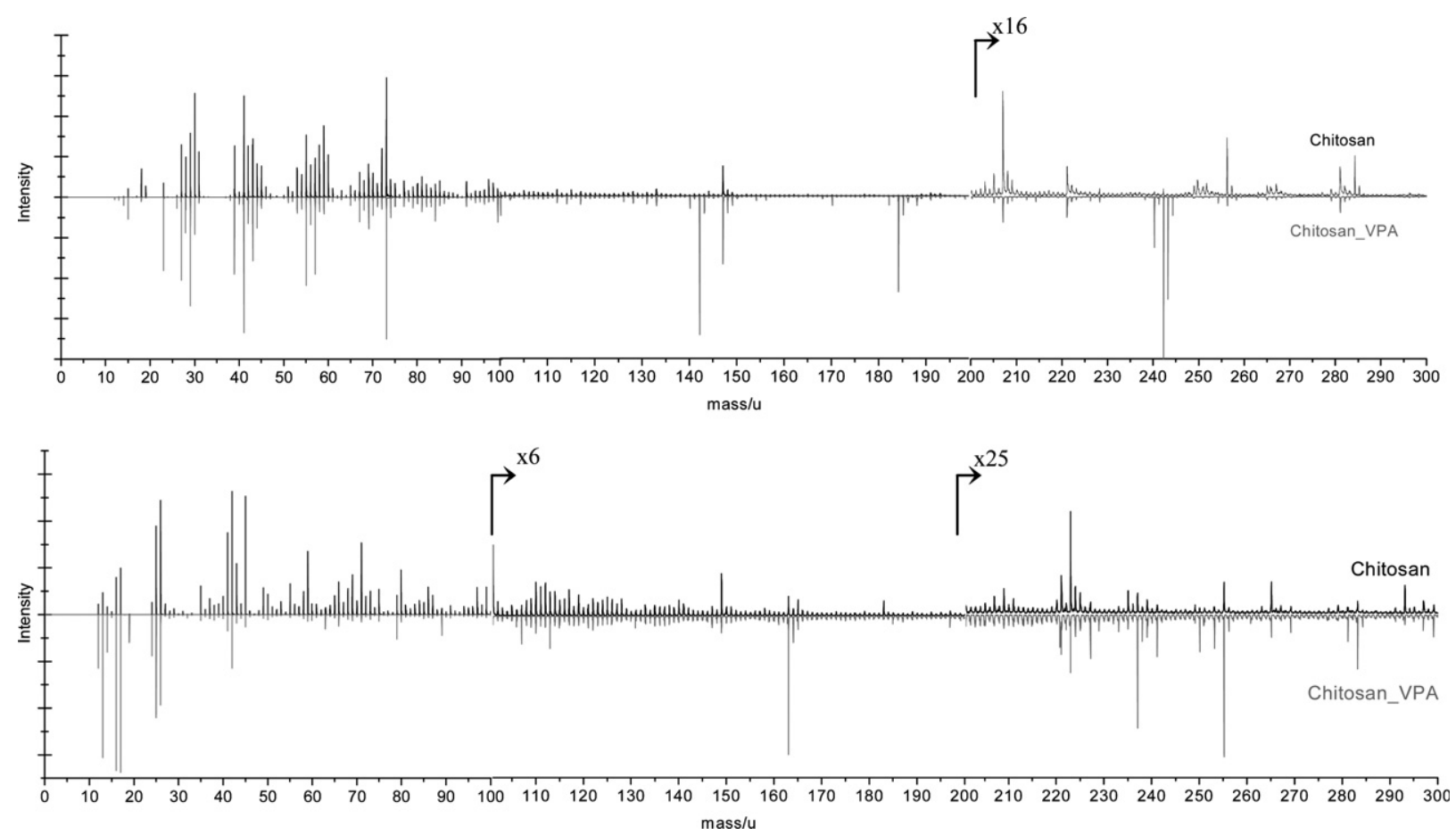

Fig. 2 Positive (top) and negative (bottom) ToF-SIMS spectra of chitosan before and after (displayed in reverse) modification with poly(vinyl phosphonic acid).
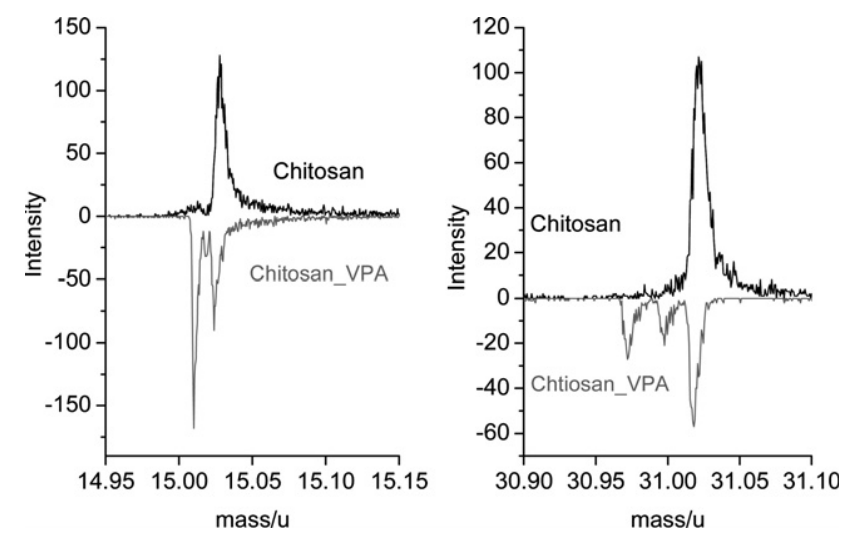

Fig. 3 High resolution ToF-SIMS, showing both higher concentration of NH (left) and presence of P (right) as a result of the applied treatment (the spectra displayed in the reverse).

topographical changes. This is especially relevant for biomaterials surfaces where topography is known to have a striking effect on biological response. ${ }^{38,39}$

Optical profiler analyses were performed in order to analyze the presence of microfeatures on the surfaces. We have detected ring-like structures on the unmodified chitosan membranes airface side. These features, with characteristic radii between 2 and $15 \mu \mathrm{m}$, disappeared after the treatment probably because of the etching process that occurs during the plasma activation (Fig. 5).

Although optical profilometry offers quick analysis of surface topography without surface contact, it is limited in lateral resolution. Scanning probe microscopy is also a non-damaging method for surface observation because of the very light forces used and extends the lateral resolution to atomic dimensions. Therefore, high resolution $\left(1 \times 1 \mu \mathrm{m}^{2}\right)$ AFM images were used to evaluate $R_{\mathrm{a}}$ (average absolute distance from average flat surface) and $R_{\mathrm{q}}$ (root mean square from average flat surface) values before and after the modification. Both surfaces showed nanopitlike textures of similar diameter and with random distribution. It could also be observed that the modification process results in a small increase of the surface roughness with changes in $R_{\mathrm{a}}$ from 1.52 to $9.13 \mathrm{~nm}$ and in $R_{\mathrm{q}}$ from 1.91 to $11.57 \mathrm{~nm}$ for chitosan and CTS-PVPA, respectively (see pictures in the ESI $\dagger$ ). These differences at the nanoscale are in agreement with previously reported $^{8,40}$ results for chitosan membranes modified by plasma and are also related with the etching process occurring during the activation step. Although the found differences in the roughness
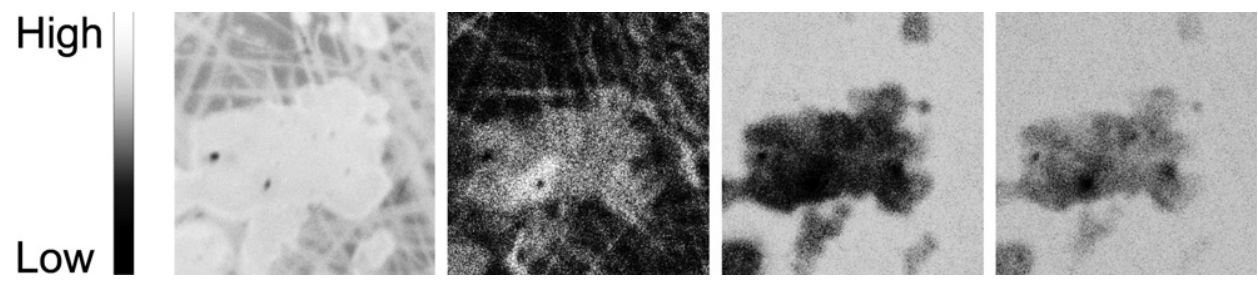

Fig. 4 Images of a $500 \mu \mathrm{m}^{2}$ area of VPA-grafted chitosan surface as reconstructed from the negative ion mass spectrum measured by static ToF-SIMS. From left to right: all ions; M59 (C2H3O2); M63 (PO2); M79 (PO3). 


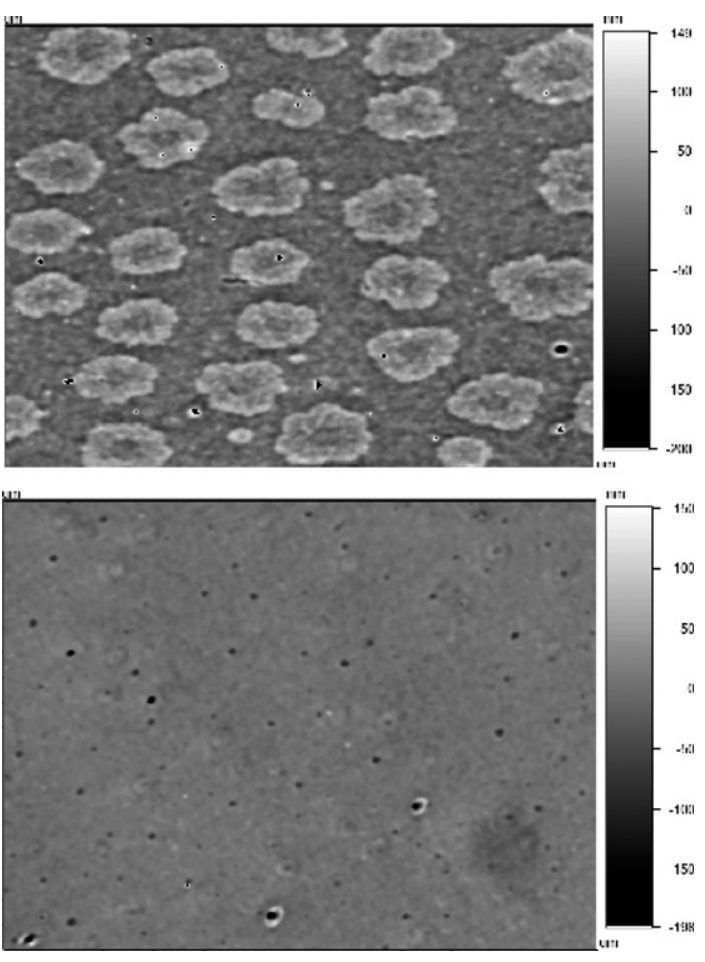

Fig. $590 \times 120 \mu \mathrm{m}^{2}$ optical profiler images of chitosan (top) and PVPA grafted (bottom) membranes. Both images were taken of the membranes face dried in contact with the air.

after PVPA grafting are small, these differences might be enough to affect cell behavior.

In their natural environment cells are in permanent contact with nano-topographic surfaces. For instance, basement membranes of various tissues are composed of pits, pores, protrusions and fibers in the range 5-200 $\mathrm{nm}$. Recent studies have shown that cells display different performances depending on the topography at the nanoscale. ${ }^{41-43}$ Lim et al. have shown that human fetal osteoblastic cells (hFOB) attachment and spreading as well as specific integrin expression were enhanced for cells cultured in 14-29 $\mathrm{nm}$ deep pits relative to flat surfaces or $45 \mathrm{~nm}$ deep pits. ${ }^{42}$

\section{Surface energy and water wettability}

Surface energy and water wettability of biomaterials significantly affect the biological process at the sub-cellular (protein adsorption) and cellular level (cell attachment, spreading, proliferation, etc.). ${ }^{37,44-47}$ The surface wettability can be directly determined by contact angle measurements, whereas a technique does not exist for direct determination of the surface energy of solids. Instead, measurements of contact angle, and several indirect empirical and semi-empirical methods can provide the information required to compute the surface energy. The calculation methods are mostly based on the assumption that the free energy of a solid surface can be split into different components representing different independent interactions. There is still an intense debate $^{27,28}$ on the applicability and correctness of using each method, as well as on the true physical meaning of some surface energy components. In this work we have calculated the surface energy both using the classical "paradigm" of Owens, Wendt,
Table 2 Equilibrium water contact angle and adhesion tension for PVPA grafted and non-treated chitosan membranes

\begin{tabular}{lll}
\hline Sample & Water contact angle ${ }^{\circ}$ & Adhesion tension $(\tau) / \mathrm{mN} \mathrm{m}^{-1}$ \\
\hline CTS & $98.5 \pm 2.5$ & $-10.8 \pm 3.1$ \\
CTS-VPA & $69.0 \pm 10.2$ & $25.7 \pm 12.2$ \\
\hline
\end{tabular}

Rabel and Kaelble (OWRK) ${ }^{25}$ and the perhaps more controversial van Oss-Chaudury-Good (vOCG) theory, ${ }^{26-28}$ which provides additional information (Lewis acid-base contributions), if taken and evaluated within the method assumptions and constraints.

Table 2 shows the water contact angle and the water adhesion tension for modified and untreated chitosan membranes. Water adhesion tension $(\tau)$, often used to predict or explain biomaterial-cell interactions as an alternative to surface energy, is defined as:

$$
\tau=\gamma_{\mathrm{w}} \cdot \cos \theta
$$

where $\gamma_{\mathrm{w}}$ is the water surface tension $\left(72.8 \mathrm{mN} \mathrm{m}^{-1}\right)$ and $\theta$ is the water contact angle. A relatively high water contact angle $\left(98.5^{\circ}\right)$ was measured for the untreated chitosan membranes. After PVPA grafting, the contact angle value decreases significantly $\left(69.0^{\circ}\right)$. Consequently, water tension adhesion values increased from -10.8 to $25.7 \mathrm{mN} \mathrm{m}^{-1}$. According to the literature, values of water tension adhesion of $30 \mathrm{mN} \mathrm{m}^{-1}$ (contact angle $65^{\circ}$ ) distinguish the hydrophobic and hydrophilic regimes. ${ }^{29,48}$ This value limits the region where long-range attractive (hydrophobic) forces become repulsive (hydration) forces. Being so, the water adhesion tension (Table 2) of untreated chitosan membranes discloses a hydrophobic surface. As expected, PVPA grafting increased the water adhesion tension as a consequence of the introduction of phosphonic groups. Nevertheless, the water adhesion tension value for PVPA grafted membranes is still in the hydrophobic regime, although within the limit where hydrophobic attraction is substituted by hydration driven repulsive forces. ${ }^{29}$

Surface energy computed using the OWRK method split the surface energy $\gamma_{\mathrm{s}}$ into a dispersive $\gamma_{\mathrm{s}}^{\mathrm{d}}$ and a polar component $\gamma_{\mathrm{s}}^{\mathrm{p}}$ according to the equation:

$$
\gamma_{\mathrm{s}}=\gamma_{\mathrm{s}}^{\mathrm{d}}+\gamma_{\mathrm{s}}^{\mathrm{p}}
$$

A high dispersive component is usually observed for polymers while the polar contribution is associated with the presence of polar groups on the surface. As shown in Table 3, the total surface energy $\left(35.6 \mathrm{mN} \mathrm{m}^{-1}\right)$ determined by the OWRK method was higher for modified membranes than for untreated material $\left(29.0 \mathrm{mN} \mathrm{m}^{-1}\right)$. The value of the polar component for unmodified chitosan was particularly low $\left(0.22 \mathrm{mN} \mathrm{m}^{-1}\right)$, thus confirming reported results for chitosan. ${ }^{7,49}$ As it was expected, the introduction of the polar phosphonic groups resulted in a drastic increase in $\gamma_{\mathrm{s}}^{\mathrm{p}}\left(8.3 \mathrm{mN} \mathrm{m}^{-1}\right)$.

In turn, according to vOCG theory, the surface energy can be calculated as a combination of dispersive and Lewis acid-base contributions:

$$
\gamma_{\mathrm{S}}=\gamma_{\mathrm{s}}^{\mathrm{LW}}+\gamma_{\mathrm{s}}^{\mathrm{AB}}
$$


Table 3 Surface Energy and its components calculated by OWRK and AB methods

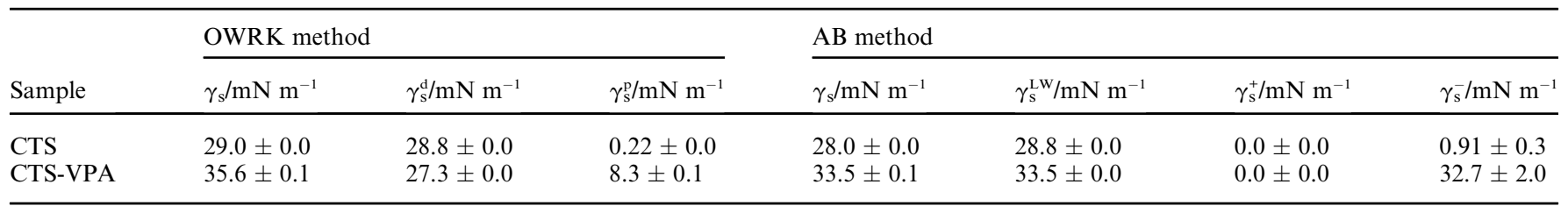

where $\gamma_{\mathrm{s}}^{\mathrm{LW}}$ is the surface energy corresponding to Lifshitz-van der Waals forces and $\gamma_{\mathrm{s}}^{\mathrm{AB}}$ describes the contribution of the acidbase interaction to the surface energy:

$$
\gamma_{\mathrm{s}}^{\mathrm{AB}}=2 \cdot\left(\gamma_{\mathrm{s}}^{-} \cdot \gamma_{\mathrm{s}}^{+}\right)^{1 / 2}
$$

where $\gamma_{s}^{-}$and $\gamma_{s}^{+}$represent, respectively, the electron donor (Lewis base) and the electron acceptor (Lewis acid) contributions or, more specifically, the particular sub-set of Lewis acid-base interactions known as hydrogen bonding.

The results obtained from the vOCG method are in accordance with the results obtained in our previous study where chitosan was functionalized with sulfonic and carboxyl groups. ${ }^{7}$ Both, untreated and modified samples presented a monopolar character with the acid component being zero (Table 3). The basic component was strongly affected by the incorporation of phosphonic groups at the surfaces, increasing from $0.91 \mathrm{mN} \mathrm{m}^{-1}$ for chitosan to $32.7 \mathrm{mN} \mathrm{m}^{-1}$ for the PVPA grafted sample. We used the scale proposed by Della Volpe and Sibone (DVB), ${ }^{28}$ who assumed a ratio for the reference water acid-base components that better describes the different "strength" of water as Lewis acid and base. By using this scale, Della Volpe and Sibone calculated the acid-base coefficients for a wide set of materials, obtaining coefficients that correctly described the chemical properties commonly expected for all tested materials. Thus, the DVB scale corrects the artifact introduced by the former vOCG scale that uses equal acid-base components for water and generates values in which all surfaces seemed to be strongly basic. This tendency has been observed even for polymer surfaces that should present pronounced Lewis acid character according to the general chemical sense. ${ }^{28}$ For this reason, we were surprised to found that, according to the calculated acid-base components, unmodified chitosan membranes presented only hydrogen bond acceptor character. This is an odd result, since hydroxyl groups can act as both a hydrogen bond donor (electron acceptor) and acceptor (electron donor).

\section{Cell behavior}

The influence of the surface modification on the biocompatibility of the studied materials was evaluated in vitro by osteoblast cell line (SaOs-2). We have observed by SEM a significant change of SaOs-2 morphology after the performed PVPA grafting (Fig. 6). While SaOs-2 cultured on untreated membranes presented a rather round shape and low adhesion during all of the studied period, cells seeded on grafted membranes showed spread morphology and higher numbers of cells attached to the surface. After one day of culture, a considerable number of adhered spread cells were observed on modified materials. In the following days the cells formed a homogeneous layer covering
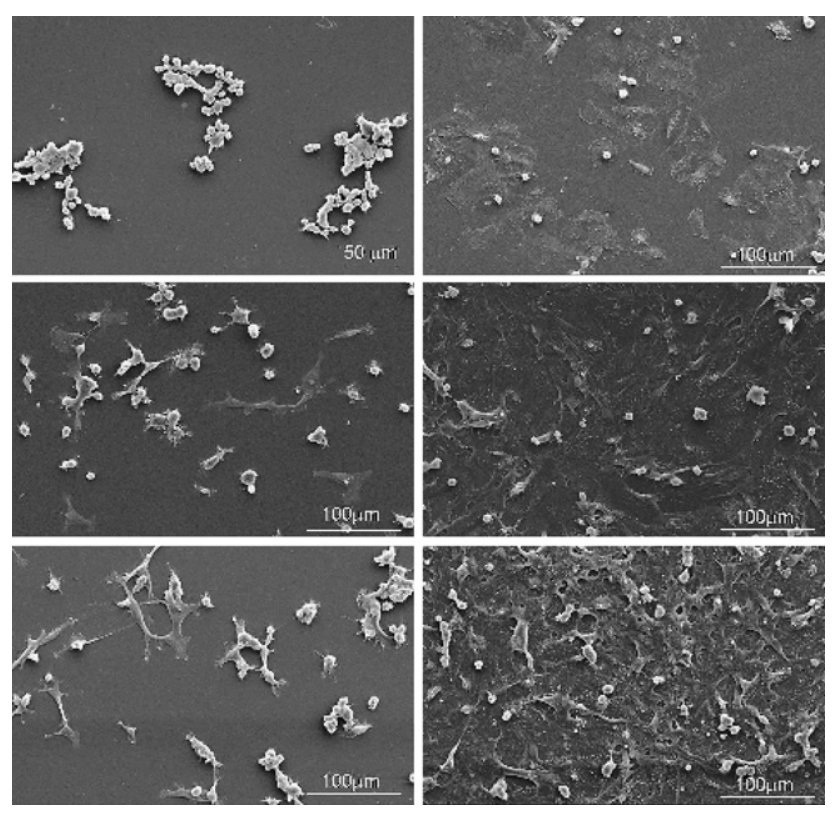

Fig. 6 SEM micrographs of SaOs-2 cultured on chitosan (left) and PVPA (right) membranes during 1, 7 and 14 days (from top to bottom).

the entire surface. In agreement with our results, for instance Lim et $a l .{ }^{47}$ reported that cytoskeletal features between osteoblastic cells cultured on hydrophilic and hydrophobic materials are notably different. Cells on hydrophilic materials presented distinct, large plaques of integrins $\left(\alpha_{\mathrm{v}}\right.$ and $\beta_{3}$ subunits) colocalized with actin stress fibers whereas there was much less development of such adhesion structures on hydrophobic surfaces.

SaOs- 2 cultured on chitosan membranes $\left(\tau=-10.8 \mathrm{mN} \mathrm{m}^{-1}\right)$ formed clump-like structures which grow in size and number with the culture time. Similar behavior has been recently reported for human foetal osteoblastic cells and three different osteoblastlike cell lines on hydrophobic substrates. ${ }^{46}$ Although the number of viable cells as indicated by Calcein AM stain increased with the culture time for both materials, the few viable cells (Fig. 7) adhered on chitosan membranes did not present the typical osteoblast-like morphology, being sparsely distributed and forming clump-like structures. On the contrary, the modified samples were covered by a homogeneous layer of viable cells.

An MTS assay (Fig. 8) showed a higher number of viable cells on PVPA grafted substrates. This difference became more evident with the increase of the culture time, indicating that the modification process has a positive effect on the long-term material response. The same tendency was observed for cell proliferation (Fig. 9). In fact, after 1 day of culture, the number of cells for PVPA grafted substrates was significantly higher than 

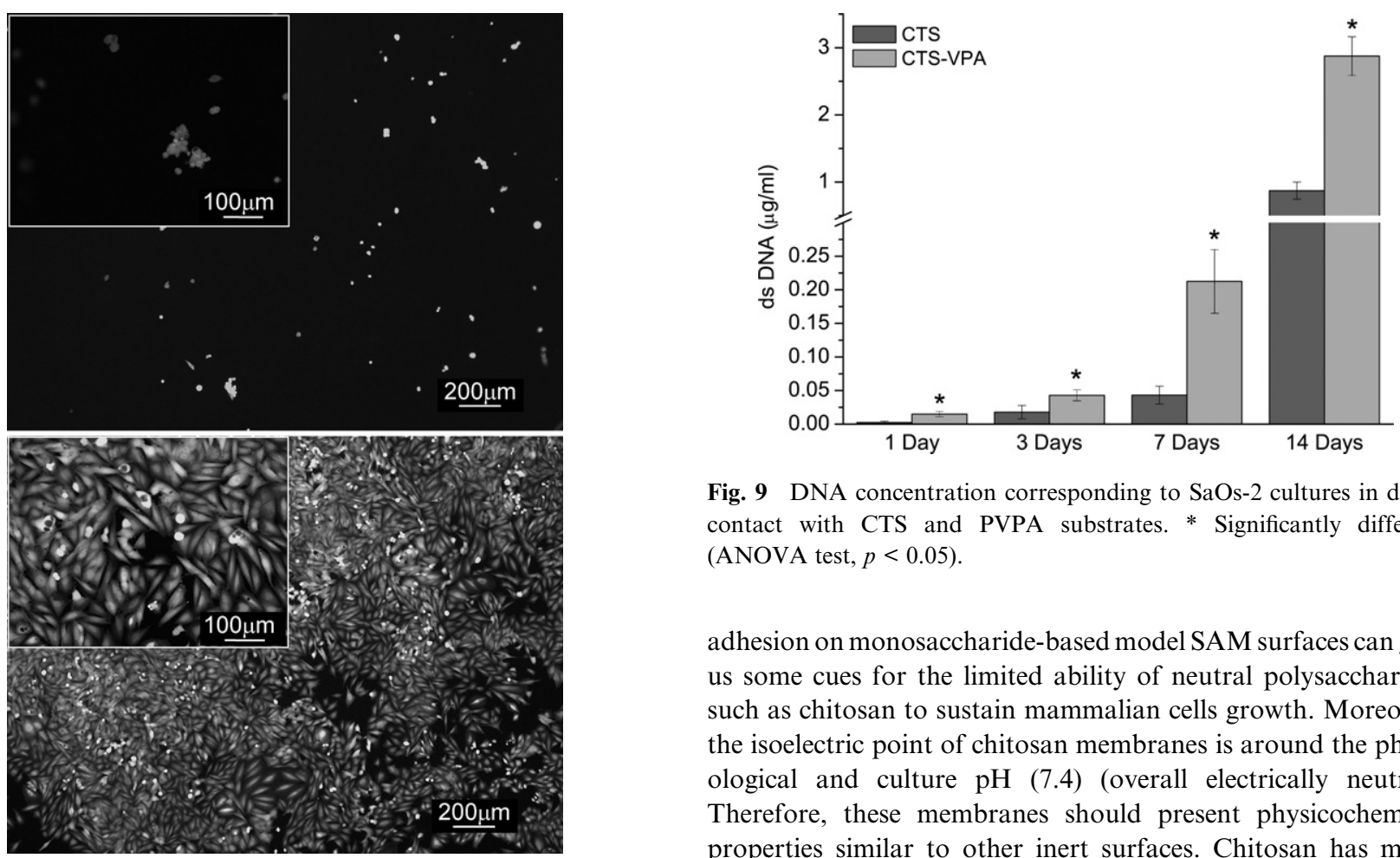

Fig. 7 Calcein AM staining of SaOs-2 cultured for 7 days on chitosan (top) and PVPA (bottom) substrates.

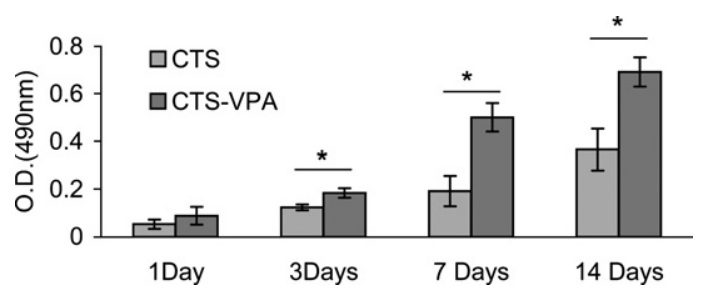

Fig. 8 Cell viability (by MTS assay) of SaOs-2 cultured on unmodified and PVPA grafted membranes.* Significantly different (ANOVA test, $p<0.05)$.

in chitosan membranes and this tendency was kept along all of the studied time periods. Therefore, it can be concluded that the grafting process of PVPA on the chitosan membranes' surface improved the osteoblast-like cells attachment, spreading, viability and proliferation.

Whitesides and co-workers examined a group of around 60 mixed self-assembled monolayers (SAMs) suggesting that functional groups that made the surfaces inert had four common features: they were (i) hydrophilic, (ii) hydrogen bond acceptors, (iii) not hydrogen bond donors, and (iv) overall electrically neutral. ${ }^{50}$ However, a few exceptions were found to this general rule; oligo(ethylene glycol) (OEG), mannitol (sugar alcohol) and maltose (disaccharide) surfaces have many hydrogen bond donors and also resisted protein adsorption and cell adhesion. ${ }^{50-52}$ Interestingly, the mannitol-presenting surface was able to resist much longer than OEG to cell adhesion on patterned surfaces. ${ }^{51}$ Although cell adhesion and proliferation is very limited on chitosan membranes, they are not fully inert because they do not inhibit those processes completely. Nevertheless, the lack of cell

Fig. 9 DNA concentration corresponding to SaOs-2 cultures in direct contact with CTS and PVPA substrates. * Significantly different (ANOVA test, $p<0.05$ ).

adhesion on monosaccharide-based model SAM surfaces can give us some cues for the limited ability of neutral polysaccharides such as chitosan to sustain mammalian cells growth. Moreover, the isoelectric point of chitosan membranes is around the physiological and culture $\mathrm{pH}$ (7.4) (overall electrically neutral). Therefore, these membranes should present physicochemical properties similar to other inert surfaces. Chitosan has many hydroxyl groups that should be able to perform both as a hydrogen bond donor and acceptor, which would include chitosan within the few exceptions to the general rule stated before. ${ }^{50}$ Nevertheless, the unexpected null value for the surface energy acid component (hydrogen bond donor) of unmodified chitosan membranes should mean that chitosan membranes' surface mainly performs as a hydrogen bond acceptor. Perhaps, this is more than a coincidence, but extending the discussion beyond this would be premature and too speculative at the moment. Finally, the cell adhesion on the treated membranes can be explained by the negatively charged nature of the grafted polymer, ${ }^{50}$ which is expected to be ionized at physiological $\mathrm{pH}$.

\section{Conclusions}

Surface chemistry characterization by XPS and ToF-SIMS indicated that chitosan membranes could be successfully grafted with poly(phosphonic acid) by plasma induced polymerization. The modification induces changes in the surface topography at the nanoscale associated with the etching process during the plasma activation step. We found that grafting of negatively charged groups such as phosphonic groups induced remarkably different osteoblast-like cell (SaOs-2) response in terms of attachment, spreading, viability and proliferation. Unmodified chitosan membranes presented very limited cell adhesion and the formation of sparsely located clump-like structures occurred for longer culture time periods. The physicochemical features of chitosan that grant its partial resistance to cell adhesion might be related to the inertness reported for other neutral saccharidebased surfaces. The plasma induced polymerization is a very simple and versatile method and it was shown to be an effective grafting methodology to render a relatively inert polysaccharide with the suitable surface properties for cell adhesion of proliferation. 


\section{Acknowledgements}

The authors acknowledge funding from EU Marie Curie Actions, Alea Jacta Est (MEST-CT-2004-008104) and Portuguese Foundation for Science and Technology (FCT) (SFRH/ BPD/34545/2007). This work was carried out under the scope of the European NoE EXPERTISSUES (NMP3-CT-2004-500283).

\section{References}

1 E. Marler and E. A. Davidson, Proc. Natl. Acad. Sci. U. S. A., 1965, 54, 648-656.

2 M. Kumar, R. A. A. Muzzarelli, C. Muzzarelli, H. Sashiwa and A. J. Domb, Chem. Rev., 2004, 104, 6017-6084.

3 R. A. A. Muzzarelli C. Muzzarelli, Chitosan chemistry: Relevance to the biomedical sciences. In Advances in Polymer Science, ed. T. T. Heinze, Springer-Verlag Berlin, Berlin, 2005, vol. 186, pp 151-209.

4 S. H. Pangburn, P. V. Trescony and J. Heller, Biomaterials, 1982, 3, $105-108$.

5 P. Sorlier, A. Denuziere, C. Viton and A. Domard, Biomacromolecules, 2001, 2, 765-772.

6 H. Y. Yeh and J. C. Lin, J. Biomater. Sci., Polym. Ed., 2008, 19, 291-310.

7 P. M. Lopez-Perez, A. P. Marques, R. M. P. da Silva, I. Pashkuleva and R. L. Reis, J. Mater. Chem., 2007, 17, 4064-4071.

8 S. S. Silva, S. M. Luna, M. E. Gomes, J. Benesch, I. Pashkuleva, J. F. Mano and R. L. Reis, Macromol. Biosci., 2008, 8, 568-576.

9 T. W. Chung, Y. F. Lu, S. S. Wang, Y. S. Lin and S. H. Chu, Biomaterials, 2002, 23, 4803-4809.

10 J. L. Cuy, B. L. Beckstead, C. D. Brown, A. S. Hoffman and C. M. Giachelli, J. Biomed. Mater. Res., 2003, 67A, 538-547.

11 J. Li, H. Yun, Y. D. Gong, N. M. Zhao and X. F. Zhang, Biomacromolecules, 2006, 7, 1112-1123.

12 R. Jayakumar, N. Nwe, S. Tokura and H. Tamura, Int. J. Biol. Macromol., 2007, 40, 175-181.

13 C.-W. Lin and J.-C. Lin, J. Biomater. Sci., Polym. Ed., 2001, 12, $543-$ 557.

14 R. Jayakumar, H. Nagahama, T. Furuike and H. Tamura, Int. J. Biol. Macromol., 2008, 42, 335-339.

15 I. F. Amaral, P. L. Granja, L. V. Melo, B. Saramago and M. A. Barbosa, J. Appl. Polym. Sci., 2006, 102, 276-284.

16 I. F. Amaral, P. L. Granja and M. A. Barbosa, J. Biomater. Sci., Polym. Ed., 2005, 16, 1575-1593.

17 R. Jayakumar, N. Selvamurugan, S. V. Nair, S. Tokura and H. Tamura, Int. J. Biol. Macromol., 2008, 43, 221-225.

18 Y. Wan, K. A. M. Creber, B. Peppley and V. T. Bui, Macromol. Chem. Phys., 2003, 204, 850-858.

19 Q. L. Li, Z. Q. Chen, B. W. Darvell, L. K. Liu, H. B. Jiang, Q. Zen, Q. Peng and G. M. Ou, J. Biomed. Mater. Res., Part B, 2007, 82B, 481-486.

20 Y. Yokogawa, K. Nishizawa, F. Nagata and T. Kameyama, J. SolGel Sci. Technol., 2001, 21, 105-113.

21 R. A. Gemeinhart, C. M. Bare, R. T. Haasch and E. J. Gemeinhart, J. Biomed. Mater. Res., Part A, 2006, 78A, 433-440.

22 J. Tan, R. A. Gemeinhart, M. Ma and W. M. Saltzman, Biomaterials, 2005, 26, 3663-3671.

23 R. M. P. da Silva, J. F. Mano and R. L. Reis, Macromol. Chem. Phys., 2008, 209, 1463-1472.
24 M. Terbojevich, A. Cosani and R. A. A. Muzzarelli, Carbohydr. Polym., 1996, 29, 63-68.

25 D. K. Owens and R. C. Wendt, J. Appl. Polym. Sci., 1969, 13, $1741-1747$

26 C. J. van Oss, M. K. Chaudhury and R. J. Good, Chem. Rev., 1988, 88, 927-941.

27 C. Della Volpe, D. Maniglio, S. Siboni and M. Morra, J. Adhes. Sci. Technol., 2003, 17, 1477-1505.

28 C. Della Volpe and S. Siboni, J. Adhes. Sci. Technol., 2000, 14, 235-272.

29 E. A. Vogler, Adv. Colloid Interface Sci., 1998, 74, 69-117.

30 D. Briggs, in Surface analysis of polymers by XPS and static SIMS, ed. D. R. Clarke, S. Suresh and I. M. Ward, Cambridge University Press, Cambridge, 1998, pp 47-87.

31 K. S. Siow L. Britcher S. Kumar H. J. Griesser, in Characterization of sulfate and phosphate containing plasma polymer surfaces, Nanoscience and Nanotechnology, 2006. ICONN '06. International Conference on, Brisbane, Australia, 3-7 July 2006, Brisbane, Australia, 2006, pp 302-305.

32 N. Adden, L. J. Gamble, D. G. Castner, A. Hoffmann, G. Gross and H. Menzel, Langmuir, 2006, 22, 8197-8204.

33 C. D. Wagner A. V. Naumkin A. Kraut-Vass J. W. Allison C. J. Powell J. R. J. Rumble, NIST Standard Reference Database 20, Version 3.5, National Institute of Standards and Technology, 2003, http://srdata.nist.gov/xps/.

34 M. Textor, L. Ruiz, R. Hofer, A. Rossi, K. Feldman, G. Hahner and N. D. Spencer, Langmuir, 2000, 16, 3257-3271.

35 S. Tosatti, R. Michel, M. Textor and N. D. Spencer, Langmuir, 2002, 18, 3537-3548.

36 A. Grenha, B. Seijo, C. Serra and C. Remunan-Lopez, J. Nanosci. Nanotechnol., 2008, 8, 358-365.

37 X. M. Liu, J. Y. Lim, H. J. Donahue, R. Dhurjati, A. M. Mastro and E. A. Vogler, Biomaterials, 2007, 28, 4535-4550.

38 K. Anselme, M. Bigerelle, B. Noel, E. Dufresne, D. Judas, A. Iost and P. Hardouin, J. Biomed. Mater. Res., 2000, 49, 155-166.

39 D. D. Deligianni, N. Katsala, S. Ladas, D. Sotiropoulou, J. Amedee and Y. F. Missirlis, Biomaterials, 2001, 22, 1241-1251.

40 A. Ogino, M. Kral, M. Yamashita and M. Nagatsu, Appl. Surf. Sci., 2008, 255, 2347-2352.

41 T. P. Kunzler, C. Huwiler, T. Drobek, J. Voros and N. D. Spencer, Biomaterials, 2007, 28, 5000-5006.

42 J. Y. Lim, A. D. Dreiss, Z. Y. Zhou, J. C. Hansen, C. A. Siedlecki, R. W. Hengstebeck, J. Cheng, N. Winograd and H. J. Donahue, Biomaterials, 2007, 28, 1787-1797.

43 J. Y. Lim, J. C. Hansen, C. A. Siedlecki, J. Runt and H. J. Donahue, J. R. Soc. Interface, 2005, 2, 97-108.

44 J. H. Lee and H. B. Lee, J. Biomater. Sci., Polym. Ed., 1993, 4, 467-481.

45 M. S. Kim, G. Khang and H. B. Lee, Prog. Polym. Sci., 2008, 33, 138 164.

46 J. Y. Lim, M. C. Shaughnessy, Z. Y. Zhou, H. Noh, E. A. Vogler and H. J. Donahue, Biomaterials, 2008, 29, 1776-1784.

47 J. Y. Lim, A. F. Taylor, Z. Y. Li, E. A. Vogler and H. J. Donahue, Tissue Eng., 2005, 11, 19-29.

48 E. A. Vogler, J. Biomater. Sci., Polym. Ed., 1999, 10, 1015-1045.

49 A. G. Cunha, S. C. M. Fernandes, C. S. R. Freire, A. J. D. Silvestre, C. Pascoal and A. Gandini, Biomacromolecules, 2008, 9, 610-614.

50 E. Ostuni, B. A. Grzybowski, M. Mrksich, C. S. Roberts and G. M. Whitesides, Langmuir, 2003, 19, 1861-1872.

51 Y. Y. Luk, M. Kato and M. Mrksich, Langmuir, 2000, 16, 9604-9608. 52 K. L. Prime and G. M. Whitesides, Science, 1991, 252, 1164-1167. 